\title{
VIGILÂNCIA DE CONTATOS DOMICILIARES DE USUÁRIOS COM HANSENÍASE MENORES DE QUINZE ANOS EM MUNICÍPIO HIPERENDÊMICO
}

\section{SURVEILLANCE OF HOME CONTACTS OF USERS WITH LEPROSY UNDER 15 YEARS OF AGE IN HYPERENDEMIC MUNICIPALITY}

\section{Débora Aparecida da Silva Santos ${ }^{1}$ * Salete Barbosa dos Santos ${ }^{2}$ Naira Rubia da Silva Ribeiro ${ }^{3}$ * Letícia Silveira Goulart ${ }^{4} *$ Magda de Mattos $^{5} *$ Lorena Araújo Ribeiro $^{6} *$ Ricardo Alves de Olinda $^{7}$}

\begin{abstract}
RESUMO
Objetivo: Analisar ações de vigilância de saúde dos contatos domiciliares de usuários com hanseníase menores de quinze anos. Métodos: Estudo de campo, transversal, norteado pela ferramenta Strengthening the Reporting of Observational Studies in Epidemiology. A amostra do estudo foi composta por 122 contatos domiciliares dos menores de quinze anos com hanseníase em Rondonópolis (MT) 2009 a 2018 selecionados de acordo com a definição do ministério da saúde de contato domiciliar. Aplicou-se questionário estruturado com variáveis sociodemográficas e ações da vigilância de contatos. A análise dos dados foi realizada por meio do Software R e testes qui-quadrado de aderência e Exato de Fisher. Resultados: Em relação às ações de vigilância prevaleceram: tempo de contato acima de dez anos (69,64\%); uma cicatriz da vacina Bacilo de Calmette-Guerin $(57,14 \%)$; não receberam a vacina $(86,61 \%)$. Quanto ao exame dermatológico, a maioria foi realizado completo $(66,07 \%)$; já o neurológico não foi realizado $(80,36 \%)$. Houve diferença estatística para todas variáveis de vigilância dos contatos domiciliares. Conclusão: Através dos dados da vigilância de contatos no município, observa-se que ainda é realizada de forma incompleta. Palavras-chaves: Hanseníase; Vigilância Epidemiológica; Saúde Pública; Menores de Idade.
\end{abstract}

\begin{abstract}
Objective: To analyze health surveillance actions of home contacts of users with leprosy under the age of fifteen. Methods: Cross-sectional field study, guided by the Strengthening the Reporting of Observational Studies in Epidemiology tool. The study sample consisted of 122 home contacts of children under fifteen with leprosy in Rondonópolis (MT) 2009 to 2018 selected according to the definition of the home contact health ministry. A structured questionnaire with sociodemographic variables and contact surveillance actions was applied. Data analysis was performed using Software R and chi-square tests of adherence and Fisher's exact test. Results: Regarding the surveillance actions, the following prevailed: contact time over ten years $(69.64 \%)$; a Bacilo de Calmette-Guerin vaccine scar $(57.14 \%)$; did not receive the vaccine $(86.61 \%)$. As for the dermatological examination, most were completed $(66.07 \%)$; the neurological was not performed $(80.36 \%)$. There was a statistical difference for all variables of surveillance of household contacts. Conclusion: Through data from the surveillance of contacts in the municipality, it is observed that it is still carried out incompletely.
\end{abstract}

Keywords: Leprosy; Epidemiological Surveillance; Public Health; Minors.

\footnotetext{
${ }^{1}$ Enfermeira, graduada em Enfermagem e Obstetrícia pela Fundação Educacional de Fernandópolis. Atualmente é professora Adjunto III do Curso de Enfermagem da Universidade Federal de Rondonópolis (UFR). Mestre em Ciências Ambientais e da Saúde pela Universidade PUC de Goiás. Doutora em Recursos Naturais pela Universidade Federal de Campina Grande (UFCG). Email: deboraassantos@hotmail.com. ORCID: https://orcid.org/0000-0003-1862-7883

${ }^{2}$ Enfermeira Graduada pela Universidade Federal de Rondonópolis. Participou de projetos de Iniciação Científica e de extensão como bolsista. E-mail: salete_venancia@hotmail.com. ORCID: https://orcid.org/0000-0002-6447-2790

${ }^{3}$ Enfermeira Graduada pela Universidade Federal de Rondonópolis. Participou de projetos de Iniciação Científica e de extensão como voluntária e do PET como bolsista. E-mail: nairarubia87@ gmail.com. ORCID: https://orcid.org/0000-0001-9584-3702

${ }^{4}$ Farmacêutica, graduada em Farmácia Análises Clínicas pela Universidade Federal de Santa Maria, Mestrado em Ciência e Tecnologia Farmacêutica pela Universidade Federal de Santa Maria. Doutorado em Biologia celular e Molecular pela Universidade Federal do Rio Grande do Sul. Atualmente é professora associada na Universidade Federal de Rondonópolis. Email: lgoulart77@yahoo.com.br. ORCID: http://orcid.org/0000-00

${ }_{5}^{5}$ Enfermeira, graduada em Enfermagem e Obstetrícia pela Universidade Federal de Santa Maria/RS. Mestrado em Enfermagem pela Faculdade de Enfermagem da Universidade Federal de Mato Grosso. Doutorado em Educação pelo Instituto de Educação da Universidade Federal de Mato Grosso. Atualmente é professora Adjunto, Nível III do Curso de Enfermagem e do Programa de Residência Multiprofissional em Saúde da Família. E-mail: magda_roo@hotmail.com. ORCID: https://orcid.org/0000-0001-83301084

${ }^{6}$ Enfermeira, graduada e mestre em enfermagem pela Faculdade de Enfermagem da UFMT (FAEN/UFMT). Doutora em Saúde Coletiva pelo Programa de Pós-Graduação em Saúde Coletiva do Instituto de Saúde Coletiva da UFMT. E-mail: lorenaenfermeira@ hotmail.com. ORCID: http://orcid.org/0000-0003-0525-4758

${ }^{7}$ Estatístico, Universidade Estadual da Paraíba, mestrado em Estatística e Experimentação Agropecuária pela Universidade Federal de Lavras. Doutor em Estatística e Experimentação Agronômica pela Universidade de São Paulo. Atualmente é professor adjunto do Departamento de Estatística, professor do quadro permanente do Mestrado em Saúde Pública da Universidade Estadual da Paraíba, professor do quadro permanente do Mestrado em Gestão e Tecnologia Ambiental da Universidade Federal de Mato Grosso-UFMT. E-mail: ricardo.estat@yahoo.com.br. ORCID: https://orcid.org/0000-0002-0509-8428
} 


\section{INTRODUÇÃO}

A hanseníase é uma doença infectocontagiosa crônica, de notificação compulsória e de investigação obrigatória, passível de cura e que acomete os nervos superficiais da pele e os troncos nervosos periféricos. Quando não tratada precocemente, pode evoluir para incapacidades físicas ${ }^{(1)}$. A transmissão é através do contato prolongado com o indivíduo portador da doença sem tratamento, pois é eliminado o bacilo Mycobacterium leprae através das vias respiratórias ${ }^{(2)}$.

Devido ao período de incubação da doença, é menos habitual acometer menores de 15 anos de idade, entretanto, em áreas endêmicas da doença, há uma incidência maior de casos nessa faixa etária ${ }^{(2)}$. A detecção de casos pediátricos indica a existência de indivíduos que não foram detectados e, consequentemente, a transmissão ativa da patologia(3).

No Brasil entre 2009 a 2018 foram notificados 311.384 casos novos de hanseníase, com taxa de detecção no ano de 2018 de 13,70 casos para 100 mil habitantes. Já no período de 2009 a 2018 foram notificados 21.808 casos em menores de quinze anos, com taxa de detecção no ano de 2018 de 3,75 para cada 100 mil habitantes ${ }^{(4)}$.

Estudo realizado em Mato Grosso no período de 2011 a 2013, destaca que houve notificação de 411 casos da hanseníase em menores de quinze anos e um coeficiente médio de 18,7/100 mil habitantes ${ }^{(5)}$. Em Rondonópolis (MT) no ano de 2010, a taxa de detecção foi de 30,02/100 mil habitantes ${ }^{(6)}$ caracterizando, assim, o município como hiperendêmico $^{(7)}$.

O MS define como contato domiciliar qualquer pessoa que habite ou tenha habitado, convive ou tenha convivido no espaço domiciliar nos últimos cinco anos anteriores ao diagnóstico da doença, independentemente de ser familiar ou não; e contato social como aqueles que de alguma forma conviveram ou convivem com relações sociais próximas e prolongadas com o contato da hanseníase não tratado; são eles vizinhos, colega de trabalho e de escola ${ }^{(2)}$.

Com a perspectiva de detecção precoce e redução dos casos de hanseníase, o MS recomenda a investigação epidemiológica de todos os contatos domiciliares e destaca como diretrizes a anamnese com enfoque aos sinais e sintomas, realização do Exame Dermatoneurológico (EDN), recomendação da vacina Bacilo de Calmette-Guerin $(\mathrm{BCG})^{(2)}$ e orientações sobre o tempo de incubação, forma de transmissão e sinais e sintomas da doença ${ }^{(8)}$.

Assim, este estudo justifica-se devido ao fato do município ser considerado hiperendêmico para a hanseníase, a escassez de estudos e a inexistência de publicações sobre o tema no município e a contribuição que o mesmo possibilitará para o 
planejamento das ações dos serviços de saúde quanto a realização da vigilância de contatos.

Diante disso a pergunta norteadora deste estudo foi: "Quais as ações de vigilância de contatos foram realizadas no município de Rondonópolis-MT’? Tal indagação levou ao objetivo do estudo cujo intuito é analisar as ações de vigilância de saúde dos contatos domiciliares de usuários com diagnóstico de hanseníase menores de quinze anos em Rondonópolis (MT) no período de 2009 a 2018.

\section{MÉTODOS}

Esta pesquisa faz parte do projeto matricial intitulado "Hanseníase: análise dos casos e da gestão do programa em um município hiperendêmico", submetido ao Comitê de Ética em Pesquisa (CEP) da Universidade Federal de Rondonópolis, por meio da Plataforma Brasil e aprovado com parecer $\mathrm{n}^{\circ} \quad 3.036 .673$ e CAAE 97441618.2.0000.8088 no dia 24 de novembro de 2018. Desta forma, respeita os aspectos éticos de pesquisa com seres humanos, de acordo com a Resolução $\mathrm{n}^{\circ}$ $466 / 2012^{(9)}$.

Estudo de campo, transversal norteado pela ferramenta $\mathrm{STROBE}^{(10)}$. Foi realizado no município de Rondonópolis, Mato Grosso (MT), relativo a série histórica de 2009 a 2018. Este município possui população estimada em 232.941 habitantes. A unidade territorial corresponde a 4.159,118 km2 e a densidade demográfica é de 47,00 hab./ $\mathrm{km}^{2(11)}$. Atualmente o município possui 42 unidades de Estratégia de Saúde da Família (ESF), 4 Centros de Saúde, 2 Policlínicas e 1 Serviço de Atenção Especializada (SAE), segundo os dados do Cadastro Nacional de Estabelecimento de Saúde (CNES) ${ }^{(12)}$.

O tipo de amostra utilizado no estudo foi amostragem não probabilística, composta por 122 contatos domiciliares dos usuários menores de quinze anos diagnosticados com hanseníase, no período de 2009 a 2018. Os critérios de inclusão foram baseados na definição de contato domiciliar recomendada pelo Ministério da Saúde ${ }^{(2)}$, considerando os contatos domiciliares de todos os casos diagnosticados com hanseníase dos menores de 15 anos no período em estudo. O critério de exclusão foi contatos domiciliares que não foram encontrados no domicílio após três tentativas.

Inicialmente foi realizada a coleta de dados secundários para identificar os usuários menores de quinze anos, através do livro de registro do Serviço de Atenção Especializada (SAE) do município.

O SAE é uma unidade de saúde referência de cunho ambulatorial de nível secundário, cujo objetivo é realizar atendimento especializado aos usuários diagnosticados com Hanseníase, Tuberculose, Microcefalia e HIV/AIDS e IST. Estes atendimentos são realizados por meio de 
encaminhamentos das unidades de Estratégia Saúde da Família (ESF) e da demanda espontânea. A equipe multiprofissional que atua no Programa da Hanseníase é composta por um enfermeiro, um médico, um técnico de enfermagem, um fisioterapeuta e uma artesã ortopédica $^{(13)}$. No livro de registro continha as seguintes variáveis: identificação do usuário, data de nascimento, idade, data de notificação, ESF de notificação, endereço, forma clínica e classificação operacional.

Posteriormente houve a coleta de dados nos prontuários dos usuários menores de quinze anos para verificação das variáveis baciloscopia e início do tratamento; e nas fichas de notificação de hanseníase foram coletadas as variáveis grau de incapacidade física e os modos de entrada e detecção da doença.

Após estas etapas, os participantes, contatos domiciliares destes usuários que tiveram diagnóstico de hanseníase, foram abordados através de visitas domiciliárias para coleta de dados primários, realizada no período de janeiro a abril de 2019, com a aplicação de um questionário estruturado, elaborado pelos pesquisadores. Foi realizado teste piloto do questionário e os pesquisadores foram previamente treinados para a aplicação do questionário, embora apresentou eficácia neste estudo o questionário não passou por processo de validação.

Os participantes foram entrevistados mediante assinatura do Termo de
Consentimento Livre e Esclarecido (TCLE) e para contatos menores de 18 anos, houve a assinatura do Termo de Assentimento Livre e Esclarecido (TALE).

As variáveis do questionário foram: dados sociodemográficos (sexo, idade, raça/cor, estado civil, escolaridade, renda familiar, ocupação, condições saneamento básico e comorbidade) e dados referentes as ações da vigilância de contatos domiciliares (tempo de contato com o usuário, classificação operacional do caso índice, cicatriz da vacina BCG, orientações sobre recomendação da vacina, exame dermatológico e neurológico, facilidade ou dificuldade na realização do exame dermatoneurológico, examinado pelo serviço de saúde, mobilização de contatos e retorno para avaliação no serviço de saúde e frequência na ESF).

A análise dos dados foi realizada utilizando frequências simples e absolutas por meio do programa Software $R$ versão 3.6.2. Para análise descritiva foram selecionadas as variáveis segundo registros de casos por ano no município em estudo e percentuais para as variáveis categóricas, organizando os dados em tabelas.

As associações entre as variáveis categóricas foram avaliadas pelo teste do quiquadrado $\left(\chi^{2}\right)$ de aderência e o teste Exato de Fisher com nível de significância estabelecido em $5 \%$ e um intervalo de confiança de $95 \%$ 
(IC95\%). Todas as análises foram efetuadas usando o software estatístico $\mathrm{R}^{(14)}$.

\section{RESULTADOS}

No período de dez anos em Rondonópolis- MT, foram notificados 87 casos de hanseníase em menores de 15 anos. estudo, que corresponderam a 112 contatos domiciliares. O ano de 2013 apresentou o maior número de contatos domiciliares (19,64\%), já 2016, o menor (1,79\%). Compara-se relação estatística entre o número de casos de hanseníase e número de contatos domiciliares (Tabela 1).

Deste total, 48 casos participaram deste

Tabela 1- Distribuição dos Casos de hanseníase em menores de 15 anos e os Contatos domiciliares de acordo com a série temporal 2009 a 2018. Rondonópolis-MT, Brasil,2019.

\begin{tabular}{|c|c|c|c|c|}
\hline \multirow[t]{2}{*}{ Ano } & \multicolumn{2}{|c|}{$\begin{array}{l}\text { Número de participantes no } \\
\text { estudo : casos de hanseníase em } \\
\text { menores de } 15 \text { anos ** }\end{array}$} & \multirow{2}{*}{$\begin{array}{c}\begin{array}{c}\text { Número de } \\
\text { contatos } \\
\text { domiciliares }\end{array} \\
\mathrm{N}(\%) \\
\end{array}$} & \multirow[t]{2}{*}{ Valor $\mathrm{p} *$} \\
\hline & $\mathrm{N}$ & $\%$ & & \\
\hline 2009 & 4 & 8,3 & $9(8,04)$ & $<0,005$ \\
\hline 2010 & 7 & 14,6 & $11(9,82)$ & \\
\hline 2011 & 6 & 12,5 & $14(12,50)$ & \\
\hline 2012 & 6 & 12,5 & $18(16,07)$ & \\
\hline 2013 & 9 & 18,8 & $22(19,64)$ & \\
\hline 2014 & 3 & 6,2 & $5(4,46)$ & \\
\hline 2015 & 6 & 12,5 & $12(10,71)$ & \\
\hline 2016 & 1 & 2,1 & $2(1,79)$ & \\
\hline 2017 & 2 & 4,2 & $6(5,36)$ & \\
\hline 2018 & 4 & 8,3 & $13(11,61)$ & \\
\hline Total & 48 & 100 & $112(100)$ & \\
\hline
\end{tabular}

*teste de qui-quadrado de aderência

Fonte: *Dados da pesquisa,2019.

Quanto as características sociodemográficas dos contatos domiciliares de hanseníase em menores de 15 anos, houve prevalência da faixa etária de 21 a 59 anos $(57,14 \%)$; raça parda $(53,57)$; escolaridade até ensino fundamental completo $(68,75 \%)$; renda familiar de 1 a 2 salários mínimos $(71,43 \%)$ e ocupação estudante/professor $(41,96 \%)$. No que se refere as condições de saneamento básico todos possuíam acesso a água potável e coleta de lixo (100\%) e em relação ao esgoto predominou fossa rudimentar $(53,57 \%)$. As variáveis idade, raça, escolaridade, renda e ocupação possuem significância estatística neste estudo $(\mathrm{p}<0,005)$ (Tabela 2$)$.

Tabela 2- Variáveis sociodemográficas e condições de saneamento dos contatos domiciliares de hanseníase de 2009 a 2018. Rondonópolis-MT, Brasil,2019.

\begin{tabular}{lll}
\hline Variáveis sociodemográficas & $\mathrm{N}(\%)$ & Valor $\mathrm{p}^{*}$ \\
\hline
\end{tabular}




\begin{tabular}{|c|c|c|c|}
\hline \multirow{3}{*}{ Idade } & 5 a 14 & $38(33,93)$ & $<0,005$ \\
\hline & 15 a 20 & $10(8,93)$ & \\
\hline & 21 a 59 & $64(57,14)$ & \\
\hline \multirow{2}{*}{ Sexo } & Feminino & $69(61,61)$ & 0,0140 \\
\hline & Masculino & $43(38,39)$ & \\
\hline \multirow{3}{*}{ Raça } & Branca & $26(23,21)$ & $<0,005$ \\
\hline & Parda & $60(53,57)$ & \\
\hline & Preta & $26(23,21)$ & \\
\hline \multirow{2}{*}{ Estado Civil } & Solteiro & $65(58,04)$ & 0,0890 \\
\hline & Casado & $47(41,96)$ & \\
\hline \multirow{2}{*}{ Escolaridade } & Analfabeto a EF completo & $77(68,75)$ & $<0,005$ \\
\hline & EM incompleto a ES completo & $35(31,25)$ & \\
\hline \multirow{3}{*}{ Renda } & < 1 salário mínimo & $10(8,93)$ & $<0,005$ \\
\hline & $>1$ até 2 salário mínimos & $80(71,43)$ & \\
\hline & 3 ou mais salários mínimos & $22(19,64)$ & \\
\hline \multirow{6}{*}{ Ocupação } & Autônomo & $15(13,39)$ & $<0,005$ \\
\hline & Estudante/Professor & $47(41,96)$ & \\
\hline & Atuação em área comercial & $7(6,25)$ & \\
\hline & Do Lar & $21(18,75)$ & \\
\hline & Motorista & $5(4,46)$ & \\
\hline & Serviços gerais & $17(15,18)$ & \\
\hline \multirow{2}{*}{$\begin{array}{c}\text { Condições de } \\
\text { Saneamento }\end{array}$} & Acesso a água potável & $112(100)$ & 0,9965 \\
\hline & Coleta de lixo & $112(100)$ & \\
\hline \multirow{2}{*}{ Esgoto } & Convencional & $52(46,43)$ & 0,4497 \\
\hline & Fossa Rudimentar & $60(53,57)$ & \\
\hline
\end{tabular}

Legenda: EF: Ensino Fundamental; EM: Ensino Médio; ES: Ensino Superior

*teste de qui quadrado de aderência

Fonte: Dados primários coletados nos domicílios dos contatos dos casos de hanseníase em menores de 15 anos em Rondonópolis (MT)

Em relação as ações de vigilância de contatos domiciliares de hanseníase em menores de 15 anos realizadas em Rondonópolis (MT) prevaleceram: tempo de contato acima de dez anos (69,64\%); classificação do caso índice multibacilar (73,21\%); uma cicatriz da vacina BCG ( $\mathrm{n}=64$; $57,14 \%$ ); não receberam orientações $(79,46 \%)$ e não receberam a vacina $(86,61 \%)$. Quanto ao exame dermatológico, a maioria foi realizado de forma completa $(66,07 \%)$; já o exame neurológico não foi realizado na maioria $(80,36 \%)$; e em relação a avaliação pela ESF e orientação de retorno para avaliação não foram realizadas $(\mathrm{n}=95 ; 84,82 \%) \quad(\mathrm{n}=104 ; \quad 92,86 \%)$ respectivamente. $\mathrm{O}$ valor-p menor que 0,005 indica alta significância estatística entre os contatos domiciliares de hanseníase dos usuários menores de 15 anos com as variáveis estudadas (Tabela 3). 
Tabela 3- Ações da vigilância de contatos domiciliares dos casos de hanseníase em menores de quinze anos no período de 2009 a 2018. Rondonópolis-MT, Brasil, 2019.

\begin{tabular}{|c|c|c|c|}
\hline \multicolumn{2}{|c|}{ Dados da vigilância de contatos } & \multirow{2}{*}{$\frac{\mathrm{N}(\%)}{5(4,46)}$} & \multirow{2}{*}{$\frac{\text { Valor } \mathrm{p}^{*}}{<0,005}$} \\
\hline \multirow{3}{*}{ Tempo de contato } & 5 anos & & \\
\hline & 06 a 10 anos & $29(25,9)$ & \\
\hline & Acima de 10 anos & $78(69,64)$ & \\
\hline \multirow{2}{*}{ Classificação dos usuários } & Multibacilar & $82(73,21)$ & $<0,005$ \\
\hline & Paucibacilar & $30(26,79)$ & \\
\hline \multirow{3}{*}{ Quantidade de cicatriz BCG } & Nenhuma & $33(29,46)$ & $<0,005$ \\
\hline & Uma & $64(57,14)$ & \\
\hline & Duas & $15(13,39)$ & \\
\hline \multirow{3}{*}{ Orientação BCG } & Sim & $17(15,18)$ & $<0,005$ \\
\hline & Não & $89(79,46)$ & \\
\hline & Não lembra & $6(5,36)$ & \\
\hline \multirow{2}{*}{ Recebeu BCG } & Sim & $15(13,39)$ & $<0,005$ \\
\hline & Não & $97(86,61)$ & \\
\hline \multirow{3}{*}{ Exame Dermatológico Realizado } & Sim, completo & $74(66,07)$ & \\
\hline & Sim, incompleto & $2(1,79)$ & $<0,005$ \\
\hline & Não & $36(32,14)$ & \\
\hline \multirow{3}{*}{ Exame Neurológico Realizado } & Sim & $14(12,50)$ & $<0,005$ \\
\hline & $\begin{array}{c}\text { Sim, não sabe se completo ou } \\
\text { incompleto }\end{array}$ & $8(7,14)$ & \\
\hline & Não & $90(80,36)$ & \\
\hline \multirow{2}{*}{ Motivo que facilitou o EDN } & Foi orientado & $19(16,96)$ & $<0,005$ \\
\hline & Vontade pessoal de realizar & $7(6,25)$ & \\
\hline \multirow{5}{*}{ Motivo que dificultou o EDN } & Não foi orientado & $80(71,43)$ & $<0,005$ \\
\hline & Família não se envolveu & $4(3,57)$ & \\
\hline & Não quis realizar & $1(0,89)$ & \\
\hline & Trabalho & $1(0,89)$ & \\
\hline & Outros & $1(0,89)$ & \\
\hline \multirow{2}{*}{ Examinado pela ESF } & Sim, sem alterações & $17(15,18)$ & $<0,005$ \\
\hline & Não & $95(84,42)$ & \\
\hline \multirow[t]{2}{*}{ Retorno para avaliação } & $\begin{array}{l}\text { Sim, em casos de sinais e } \\
\text { sintomas }\end{array}$ & $8(7,14)$ & $<0,005$ \\
\hline & Não & $104(92,86)$ & \\
\hline \multirow{3}{*}{ Frequência na ESF } & Sim/Últimos 6 meses & $62(55,36)$ & $<0,005$ \\
\hline & Sim/Últimos 12 meses & $16(14,29)$ & \\
\hline & Não & $34(30,36)$ & \\
\hline
\end{tabular}

Legenda: EDN: Exame Dermatoneurológico; ESF: Estratégia de Saúde da Família

Fonte: Dados primários coletados nos domicílios dos contatos dos casos de hanseníase em menores de 15 anos em Rondonópolis (MT).

\section{DISCUSSÃO}

Em Rondonópolis (MT) no período do estudo, os 48 casos participantes da pesquisa corresponderam a 112 contatos domiciliares, com média de 2,54 contatos para cada caso de hanseníase. Dados semelhantes do município de Buriticupu (MA) no ano de 2007, cujos casos índices possuíam de três a cinco contatos $(38,4 \%)^{(15)}$. 
Ressalta-se que nos achados do município de Rondonópolis (MT) a variável idade e raça foram estatisticamente significantes, já o mesmo não pôde ser observado em relação ao sexo.

Em relação a escolaridade dos contatos domiciliares deste estudo há predomínio da baixa escolaridade, corroborando com $46,7 \%$ dos casos no estado do Pará (2012 a 2015), destacando que o baixo nível de escolaridade é fator de risco para a hanseníase para os contatos domiciliares e que pode estar relacionado ao não comparecimento para avaliação e limitação do entendimento sobre o controle da doença ${ }^{(16)}$.

Os achados deste estudo ainda demonstram que a maioria dos contatos possuem baixa renda e, apesar de boas condições de saneamento nas residências, grande parte possui fossa rudimentar, evidenciando um índice elevado de vulnerabilidade social. Araújo e Lana ${ }^{(17)}$ destacam que situação de pobreza e desigualdade social são fatores contribuintes para a manutenção da cadeia de transmissão da hanseníase.

No presente estudo o tempo de contato entre os usuários com hanseníase menores de 15 anos e seus contatos domiciliares foram superiores a 10 anos $(69,64 \%)$. Considerando o período de incubação da hanseníase, estes contatos possuem maiores chances de adquirirem a doença devido contato prolongado com o caso índice ${ }^{(2)}$.

No que se refere a classificação operacional, a prevalência neste estudo são contatos de casos multibacilares. Considerando que esta classificação é a forma infectante da doença e que o domicílio é uma fonte significativa de transmissão, os contatos domiciliares destes usuários possuem intenso fator de risco para adquirir a doença ${ }^{(2)}$.

Com relação a cicatriz vacinal da BCG, a prevalência neste estudo é de indivíduos que possuem uma cicatriz (57,14\%). Cabe ressaltar que o Ministério da Saúde recomenda a aplicação da vacina BCG em todos os contatos domiciliares da hanseníase sem sinais e sintomas, o indivíduo que tem apenas uma cicatriz deverá receberá uma dose da vacina ${ }^{(7)}$, entretanto, observa-se que neste estudo a maioria não recebeu a dose da vacina $(86,61 \%)$ e nem orientações a respeito da mesma $(79,46 \%)$, acarretando assim em uma vigilância epidemiológica de contatos incompleta.

Através deste estudo pode-se identificar que na maioria dos contatos domiciliares foram realizados o exame dermatológico $(66,07 \%)$, já o exame neurológico não foi realizado $(80,36 \%)$. O MS destaca que o diagnóstico da hanseníase é principalmente clínico e epidemiológico e que a finalidade da vigilância de contatos é realizar a busca de novos casos através do exame dermatoneurológico, porém, a 
realização forma incompleta contribui para a permanência da cadeia de transmissão da doença $^{(2)}$.

O motivo facilitador e dificultador quanto a realização do exame dermatoneurológico foi a orientação $(16,93 \%)$ e a falta desta $(71,43 \%)$. As ações realizadas pelas unidades de saúde como a análise dos indicadores epidemiológicos da doença, a realização da educação em saúde com intuito de dar autonomia aos usuários da hanseníase e aos seus contatos são meios que podem colaborar para uma atuação profissional resolutiva $^{(18)}$.

A maioria dos participantes desta pesquisa não foram examinados na ESF $(84,82 \%)$. Neste sentido, em virtude de que o diagnóstico de casos novos da hanseníase em menores de 15 anos é um possível indicador que ainda há casos não diagnosticados e desassistidos pelas unidades de saúde, faz se necessário realizar a busca ativa através do exame de contato domiciliar para que haja redução da cadeia de transmissão da doença $^{(19)}$. Em razão de que a Atenção Primária a Saúde (APS) possui o objetivo de desenvolver um cuidado integral que impacte positivamente na situação de saúde da coletividade, e que o exame de contatos é uma estratégia de suma importância para redução dos níveis de endemicidade da doença ${ }^{(20)}$, este dado reflete uma assistência inoperante neste município.

\section{CONCLUSÃO}

Através dos dados da vigilância de contatos no município do estudo observa-se que a mesma ainda é realizada de forma incompleta, o que reforça a necessidade de treinamentos e capacitações de forma contínua aos profissionais da saúde, em especial, aqueles da atenção primária, considerando que a APS é porta de entrada a mesma deve ser e estar apta a realizar as atividades que contribuem para a redução da carga da hanseníase no país. Por fim, este estudo poderá contribuir de forma positiva com a prática dos profissionais de saúde atuantes nas instituições responsáveis pela vigilância da hanseníase, norteando-os na tomada de decisão de estratégias para redução e controle da cadeia de transmissão da hanseníase no município.

\section{REFERÊNCIAS}

1. Brasil. Ministério da Saúde. Secretaria de Vigilância em Saúde. Departamento de Vigilância das Doenças Transmissíveis. Guia prático sobre a hanseníase [Internet]. Brasília: Ministério da Saúde 2017 [Acesso em 15 de Jan 2020] Disponível em:

http://bvsms.saude.gov.br/bvs/publicacoes /guia_vigilancia_saude_volume_2.pdf

2. Brasil. Ministério da Saúde. Secretaria de Vigilância em Saúde. Departamento de Vigilância das Doenças Transmissíveis. Guia prático sobre a hanseníase [Internet]. Brasília: Ministério da Saúde 2019 [Acesso em 21 de Jan 2020] Disponível em:

https://portalarquivos2.saude.gov.br/imag 
es/pdf/2019/junho/25/guia-vigilanciasaude-volume-unico-3ed.pdf

3. Oms. Organização Mundial da Saúde. Estratégia Mundial de eliminação da lepra 2016:2020: Acelerar a ação para um mundo sem lepra. OMS 2016[Internet]. [Acesso em 21 Set 2019] Disponível em: http://nhe.fmrp.usp.br/wpcontent/uploads/2017/06/Hanseniase_201 6-2020.pdf

4. Brasil. Ministério da Saúde. Secretaria de Vigilância em Saúde. Boletim Epidemiológico da Hanseníase [Internet]. 2020 [Acesso em 04 Mai 2020]; número especial:1-51.Disponível em: https://www.saude.gov.br/images/pdf/202 0/janeiro/31/Boletim-hanseniase-2020web.pdf

5. Freitas BHBM, Xavier DR, Cortela DCB, Ferreira SMB. Hanseníase em menores de quinze anos em municípios prioritários, Mato Grosso, Brasil. Rev. bras. epidemiol [Internet]. 2018 [Acesso em 2019 Nov 9];21(e180016):1-12. DOI10.1590/1980549720180016. Disponível em: http://www.scielo.br/scielo.php?script=sci _arttext\&pid=S1415790X2018000100414 $\& \operatorname{lng}=$ en $\&$ nrm $=$ iso

6. Santos DAS, Santos SB, Ribeiro NRS, Goulart LS, Olinda RA. Tendência dos casos de hanseníase em menores de quinze anos em Rondonópolis-MT. Revista O mundo Saúde [Internet]. 2018 [Acesso em 20 de Nov 2019];42(3):609627. Disponível em: https://pesquisa.bvsalud.org/portal/resourc e/pt/mis-40159.

7. Brasil. Ministério da Saúde. Secretaria de Vigilância em Saúde. Diretrizes para vigilância, atenção e eliminação da hanseníase como problema de saúde pública: manual técnico operacional[Internet]. 2016 [Acesso em 20 de Nov 2019]. Disponível em: https://www.saude.gov.br/images/pdf/201 6/fevereiro/04/diretrizes-eliminacaohanseniase-4fev16-web.pdf

8. Brasil. Ministério da Saúde. Gabinete do Ministro. Portaria $\mathrm{n}^{\circ} 3125$, de 7 de outubro de 2010. Aprova as diretrizes para vigilância, atenção e controle da hanseníase. Diário Oficial da República Federativa do Brasil, Poder Executivo[Internet]. Brasília, DF. 15 Out. 2010 [Acesso em 15 de Nov 2019]; Seção 1, p.55. Disponível em: https://bvsms.saude.gov.br/bvs/saudelegis /gm/2010/prt3125_07_10_2010.html

9. Brasil. Ministério da Saúde. Resolução ${ }^{\circ}$ 466, de 12 de dezembro de 2012. [Internet]. Brasília, DF. 12 Dez. 2012 [Acesso em 15 de Jan 2020]. Disponível em:

https://bvsms.saude.gov.br/bvs/saudelegis/ cns/2013/res0466_12_12_2012.html

10. Equator. Enhancing the QUAlity and Transparency Of health Research[Internet]. 2020 [cited 2020 mai 06]; Disponível em: https://www.equatornetwork.org/reporting-guidelines/strobe/

11. Ibge. Instituto Brasileiro de Geografia e Estatística [Internet]. 2020 [Acesso em 12 de Fev 2020]; Disponível em: https://cidades.ibge.gov.br/

12. Cnes. Cadastro Nacional do Estabelecimento de Saúde [Internet]. 2020 [Acesso em 10 de Fev 2020]; Disponível em:

http://cnes.datasus.gov.br/pages/estabeleci mentos/consulta.jsp

13. Sae. Serviço de Atenção Especializada. Manual: regimento interno, normas e rotinas e protocolos operacionais padrão. Rondonópolis, 2017; 97p.

14. R Core Team (2020). R: A language and environment for statistical computing. $\mathrm{R}$ Foundation for Statistical Computing, Vienna, Austria. URL https://www.Rproject.org/. 
15. Leite KKC, Costa JML, Barral A, Caldas AJM, Corrêa RGCF, Aquino DMC. Perfil epidemiológico dos contatos de casos de hanseníase em área hiperendêmica na Amazônia do Maranhão. Caderno de Saúde Coletiva, Rio de Janeiro [Internet]. 2009[Acesso em 05 de mai 2020];17,1:235-249. Disponível em: http://www.cadernos.iesc.ufrj.br/cadernos/ images/csc/2009_1/artigos/Art_16CSC09 _1.pdf

16. Cunha MHCM, Silvestre MPSA, Silva AR, Rosário DDS, Xavier MB. Fatores de risco em contatos intradomiciliares de pacientes com hanseníase utilizando variáveis clínicas, sociodemográficas e laboratoriais. Revista Pan-AmSaúde[Internet]. 2017[Acesso em 05 de mai 2020];8,2:23-30. DOI: $10.5123 / \mathrm{S} 2176-62232017000200003$

Disponível em: http://scielo.iec.gov.br/scielo.php?script=s ci_arttext\&pid=S217662232017000200021

17. Araújo KMFA, Lana FCF. Relação da hanseníase com a cobertura da estratégia saúde da família e condições socioeconômicas. Ciencia Y Enfermeria 2020[Internet]. [Acesso em 05 de mai 2020];26,1:9. Disponível em: https://scielo.conicyt.cl/pdf/cienf/v26/071 7-9553-cienf-26-1.pdf

18. Lozano AW, Neto JMP, Femina LL, Donda P, Silva CFG, Nardi ST, Paschoal VDA. O domicílio como importante fator de transmissão da hanseníase. Rev Enferm UFPE [Internet]. 2019[Acesso em 29 de abr 2020];13 e:241790:1-10. DOI: 10.5205/1981-8963.2019.241790.

Disponível em: https://www.leprosyinformation.org/resource/o-domiciliocomo-importante-fator-de-transmissao-dahanseniase

19. Freitas BHBM, Cortela DCB, Ferreira SMB. Tendência da hanseníase em menores de 15 anos em Mato Grosso
(Brasil), 2001-2013. Revista de Saúde Pública [Internet]. 2017[Acesso em 29 de abr 2020];51,28: 1-10. DOI: doi.org/10.1590/S1518-

8787.2017051003884. Disponível em: https://www.scielo.br/pdf/rsp/v51/pt_0034 -8910-rsp-S151887872017051006884.pdf

20. Brasil. Ministério da Saúde. Portaria $n^{\circ}$ 2.436, de 21 de setembro de 2017. Aprova a Política Nacional de Atenção Básica, estabelecendo a revisão de diretrizes para a organização da Atenção Básica, no âmbito do Sistema Único de Saúde (SUS). [Internet]. Brasília, DF. 21 Set. 2017b [Acesso em 23 de abr 2020]; Disponível em:

https://bvsms.saude.gov.br/bvs/saudelegis/ gm/2017/prt2436_22_09_2017.html.

Submissão: 2020-08-08

Aprovado: 2021-02-07 